Service social

\title{
L'aide par les pairs et les groupes d'entraide comme solutions de rechange et comme compléments à l'aide professionnelle
}

\section{Gary A. Lloyd}

Volume 38, numéro 1, 1989

Aspects psychosociaux du Sida

URI : https://id.erudit.org/iderudit/706419ar

DOI : https://doi.org/10.7202/706419ar

Aller au sommaire du numéro

Éditeur(s)

École de service social de l'Université Laval

ISSN

1708-1734 (numérique)

Découvrir la revue

Citer cet article

Lloyd, G. A. (1989). L'aide par les pairs et les groupes d'entraide comme solutions de rechange et comme compléments à l'aide professionnelle. Service social, 38(1), 8-16. https://doi.org/10.7202/706419ar
Résumé de l'article

Compte tenu que le nombre de personnes ayant une formation professionnelle ne sera jamais suffisant pour affronter la crise du VIH, on doit trouver des solutions de rechange. Deux types de ressources sont ici proposées : l'aide par les pairs et les groupes d'entraide. La mise en place de ces formes d'aide exige une formation appropriée. Elles peuvent être des compléments essentiels aux stratégies de prévention, de contrôle et d'aide, et en ce sens, elles devront être intégrées à l'ensemble du système de soins. 
LLOYD, Gary A., professeur à l'École de service social et coordonnateur à l'Institute for Research and Training in HIV/ AIDS Counseling, Tulane University, Nouvelle Orléans, Louisiane.

\section{L'aide par les pairs et les groupes d'entraide comme solutions de rechange et comme compléments à l'aide professionnelle *}

Il a été clairement démontré que la pandémie d'infection VIH (virus d'immunodéficience humaine) et de sida a des conséquences sérieuses sur le développement économique et social, sur l'allocation des ressources et sur la stabilité et la cohésion politiques dans les régions où leur fréquence est élevée. Même aux endroits où le nombre de personnes infectées ou atteintes est moins élevé, la stigmatisation et la peur liées au VIH et au sida peuvent conduire à une utilisation peu judicieuse des minces ressources ou mener à des mesures répressives pouvant aller jusqu'à des programmes de dépistage obligatoire. Les systèmes de soins de santé - déjà surchargés dans la plupart des parties du monde - les formes traditionnelles de soins des malades et des mourants et les organismes de soutien à la famille sont mis à l'épreuve par le nombre de personnes qui requièrent de l'aide pour faire face à une infection incurable ou à une maladie mortelle.

On doit reconnaître que sans tenir compte de la portée ou du succès de l'éducation dans le domaine de la santé et de la promotion de la santé et des programmes de prévention, le nombre de travailleurs sociaux et de conseillers qui ont une formation professionnelle ne sera jamais suffisant pour affronter la crise du VIH. On doit donc trouver des solutions de rechange. L'expérience de l'Amérique du Nord et de l'Europe démontre qu'en ce domaine, deux types de ressources sont valables : l'aide par les pairs et les groupes d'entraide. Il reste à évaluer si 
de tels substituts à la consultation psychosociale officielle sont possibles et satisfaisants dans les autres parties du monde et à l'intérieur d'autres cultures.

Puisque la prévention, le contrôle et le soutien sont les seules méthodes qui, présentement, permettent d'affronter la pandémie de $\mathrm{VIH}$, on ne doit pas s'étonner que les professionnels de la santé recherchent des moyens d'augmenter la capacité existante des systèmes de soins de santé pour faire face à la crise. Cette croissance a dû être entreprise dans bon nombre de systèmes où le financement, insatisfaisant et inconsistant, ne permet même pas de fournir les soins de base ou de routine. Ces systèmes ne sont pas dotés de personnel formé en promotion de la santé ou en méthodes de relation d'aide et de plus, ne semblent pas susceptibles de recevoir une aide additionnelle du gouvernement central pour relever le défi du VIH et du sida.

En Amérique du Nord et en Europe, l'aide par les pairs et les groupes d'entraide ont été utilisés à titre de moyens rentables et efficaces pour compléter le traitement et les soins fournis par le système officiel et les familles. Mises sur pied dans les communautés homosexuelles - où elles se multiplient encore - ces deux formes d'aide sont maintenant intégrées au système global et fournissent des soins à tous ceux qui en ont besoin : les personnes infectées ou atteintes du sida, celles qui s'inquiètent de l'être et les proches qui ont tous en commun une préoccupation pour le VIH et le sida. Ces méthodes connaissent un tel succès que certaines personnes les perçoivent comme substituts à l'aide professionnelle. II serait plus utile de les définir comme des variantes ou des solutions de rechange à l'aide formelle ou professionnelle qui, dans certains cas, devrait être la méthode retenue.

Mis à part les avantages évidents que représentent l'expansion des réseaux et l'augmentation des ressources axées sur le traitement, l'aide par les pairs et les groupes de soutien ou d'entraide constituent une facette unique et importante de l'effort global axé sur la relation d'aide. Ces méthodes constituent une force importante puisque, à un degré impossible à atteindre par les aidants professionnels, le pair - et habituellement le responsable du groupe d'entraide - a déjà vécu les mêmes expériences: l'aidant et l'aidé partagent une caractéristique importante, soit la séropositivité ou la maladie. Ils peuvent intervenir de façon plus directe sur les sentiments d'isolement, de peur et de désespoir, aider à mobiliser les ressources internes et externes et offrir leur soutien pour permettre aux autres de vivre avec le VIH ou le sida.

Tandis que les possibilités de ces deux méthodes ont été démontrées en Europe et en Amérique du Nord, peu d'expériences prouvent qu'elles seront acceptées et utiles en d'autres parties du monde. À cause des interdits culturels de parler des problèmes personnels à 
l'extérieur de la famille, des tabous liés aux discussions ouvertes sur le sexe et la sexualité, des tensions et des diversités ethniques, et des rôles rigides souvent imposés aux groupes non professionnels, l'aide par les pairs et les groupes d'entraide peuvent être perçus comme menaçants ou inappropriés aux personnes qui souffrent de VIH ou de sida ou aux différents intervenants. Le système médical officiel, principal pourvoyeur et coordonnateur de services, peut considérer ces méthodes comme des attaques à son endroit. II peut aussi être nécessaire d'introduire, dans le système officiel de soins de santé, une forme d'aide psychosociale culturellement acceptable avant que les médecins, les hommes politiques, les patients et les familles approuvent des méthodes moins formelles. Pour une mise en place réussie de ces méthodes dans de nouveaux milieux, il sera sûrement nécessaire, à toutes les étapes de la planification de programmes nationaux et de projets à moyen terme, d'inclure une formation et un plan d'application de services d'aide psychosociale professionnelle à la problématique du VIH et du sida, et de prévoir un apport financier à l'aide par les pairs et aux groupes d'entraide.

\section{Buts de l'aide par les pairs et des groupes d'entraide}

L'aide par les pairs et les groupes d'entraide peuvent compléter les services de soins médicaux. Les avantages qu'ils présentent ne sont pas limités à l'expansion du réseau de services et à l'utilisation de ressources humaines encore inexploitées, mais ils permettent de faire appel à l'expérience personnelle de façon productive pour la société. Par exemple, l'aidant séropositif (qui a dû affronter les conséquences de son état) peut être plus en mesure d'aider une autre personne à comprendre et à réagir à un test positif. Les principaux buts de l'aide par les pairs sont les suivants : partager une expérience personnelle, diminuer les sentiments d'isolement et d'aliénation et fournir un soutien social. Les buts plus spécifiques sont : 1) d'augmenter l'étendue des ressources en développant et en soutenant les systèmes non officiels d'aide psychosociale ; 2) d'engager, dans les soins à autrui, les personnes qui ont déjà vécu les conséquences émotionnelles d'un diagnostic et connu des sentiments de vulnérabilité ; 3) d'aider toutes les personnes concernées à maintenir des sentiments d'autonomie et de contrôle aussi longtemps qu'il se peut, de façon réaliste ; et 4) de fournir une source d'information à jour et un soutien psychosocial. Les personnes qui peuvent atteindre ces buts sont celles qui ont dû, par le passé, affronter et réagir à la situation que la personne aidée doit vivre dans le moment.

Les groupes d'entraide partagent le même but que l'aide par les pairs même si, par définition, ils donnent une plus grande priorité à un 
soutien émotionnel à la fois mutuel et pertinent. On émet l'hypothèse que toute personne infectée par le VIH connaîtra, jusqu'à un certain point, des sentiments de colère et de dépression, de peur du rejet, de stigmatisation et d'isolement social réel ou supposé. Chacune de ces réactions peut être atténuée par un engagement avec d'autres personnes qui ressentent ou ont ressenti de tels sentiments et qui ont trouvé différents moyens d'y faire face.

L'avantage de l'aide par les pairs et des groupes d'entraide, lorsqu'ils sont adaptés au contexte culturel, est de constituer des regroupements où les participants peuvent partager des expériences personnelles relatives à une infection ou une maladie qui menace leur vie. Les relations entre des personnes qui vivent des expériences semblables peuvent faciliter la personnalisation de l'information sur la santé, le soutien nécessaire à la modification des comportements à risques, l'entretien des contacts sociaux et économiques le plus longtemps possible, le passage des étapes de douleur et de deuil et la planification de l'avenir. D'autres formes d'aide peuvent offrir chacun de ces avantages mais le partage d'expériences communes confère une force particulière à l'aide par les pairs et aux groupes d'entraide.

\section{Qui peut être conseiller ou responsable de groupe?}

II n'est pas nécessaire que les conseillers, dans une aide par les pairs, et les responsables de groupes d'entraide aient une formation professionnelle. Cependant, après avoir été sélectionnés, ils doivent recevoir une formation spéciale avant qu'on leur confie la responsabilité d'intervenir dans la vie d'autres personnes.

Pour répondre au premier critère de sélection, le conseiller ou le responsable doit être lui-même infecté par le VIH ou atteint du sida. Le candidat doit, selon le deuxième critère, avoir atteint un degré d'acceptation ou canalisé sa colère pour assurer l'écoute attentive d'une autre personne sans lui imposer son propre point de vue et sans établir de comparaisons entre leurs réactions propres. Ce critère peut être difficilement applicable dans des milieux culturels où les conseils et les directives autoritaires sont des caractéristiques inhérentes aux systèmes de services sociaux et de santé.

Le troisième critère porte sur l'obligation à la plus entière confidentialité de la part du conseiller ou responsable. Ce dernier doit comprendre et agir en conformité avec ce principe tout au long du processus de consultation et doit être en mesure d'enseigner aux autres la façon de tenir les discussions de groupe confidentielles. Si l'on ne peut être en droit de s'attendre à ce type de confidentialité, si l'on ne 
peut l'enseigner et si, pour des raisons organisationnelles, culturelles ou sociales, on ne peut l'appliquer, l'aide par les pairs et les groupes d'entraide ne pourront alors être partie intégrante d'une stratégie de prévention et de contrôle du sida.

\section{Formation des conseillers et des responsables}

Il est entendu que les conseillers et les responsables de groupes d'entraide doivent recevoir une formation avant de pouvoir occuper ces postes. Qui fournira cette formation ? Puisque la relation d'aide a en soi un caractère novateur en plusieurs endroits, la plupart des programmes de VIH/SIDA seront intégrés au secteur formation d'un programme plus global de prévention et de contrôle. Dans la plupart des cas, l'infrastructure de services officiels de relation d'aide est peu développée ou inexistante. Les ressources professionnelles disponibles sont susceptibles d'être surexploitées. À peu près partout dans le monde, cette situation signifie que la formation des conseillers ou responsables de groupes d'entraide sera dispensée par des individus qui ont participé à un atelier du programme global sur le sida (et qui ne se sentent peutêtre pas encore très sûrs d'eux comme professeurs), ou qui ont acquis une connaissance de l'aide psychosociale pendant leur formation en service social ou dans une autre discipline (et qui devraient ajouter cette formation à un calendrier professionnel déjà bien rempli). Quelle que soit la méthode choisie, cette formation doit être dispensée en priorité. Il est souvent difficile de le faire puisque d'autres besoins de formation - par exemple la nécessité de test avant et après l'aide apportée - peuvent sembler plus urgents ou lorsqu'une telle formation s'ajoute aux lourdes tâches déjà confiées à certaines personnes.

Les responsables de l'inclusion de ces méthodes dans une stratégie globale d'aide psychosociale devraient établir des critères de sélection qui s'ajoutent à ceux qui ont déjà été mentionnés, critères qui permettraient de traiter de problèmes culturels et de particularités liées au milieu. Le point principal est l'importance accordée au recrutement des candidats, au choix et à l'évaluation de ces derniers et à la sélection selon des caractéristiques personnelles. On doit garder à l'esprit que le partage d'expériences communes peut apporter un soulagement et fournir un soutien mais il peut aussi, au contraire, être destructeur et nuisible s'il est fait sans une direction appropriée et satisfaisante. Si, par exemple, un conseiller qui n'a pas dépassé le stade du " pauvre moi » est assigné à une personne qui affiche une attitude de retrait, ce conseiller contribuera à augmenter plutôt qu'à diminuer les tensions et les sentiments négatifs. Cette observation relève de l'évidence mais elle doit 
toutefois être faite. On sera tenté, avec l'augmentation des demandes, de faire ouvrer des conseillers qui n'ont pas encore atteint le degré nécessaire de préparation. II serait prudent d'utiliser les formes de soutien offertes par des groupes religieux et éducatifs ou des institutions si la formation n'est pas dispensée.

En résumé, l'aide par les pairs et les groupes d'entraide ne peuvent fonctionner sans que l'on ait l'assurance que les conseillers et les responsables aient reçu une formation satisfaisante. Cette formation doit comporter : 1) une présentation et une exploration du concept de conscience de soi et de son importance ; 2 ) une exploration des formes de soutien ou des barrières au partage d'informations personnelles avec des personnes étrangères à la famille ; 3 ) les principes de base en dynamique de groupe ; 4) les méthodes visant le comportement et le contrôle des émotions ressenties à l'écoute d'expériences semblables vécues par une autre personne ; et 5) les façons culturellement acceptables de fournir des conseils dont la contrepartie est la compréhension d'un besoin d'objectivité et d'empathie.

\section{Confidentialité}

Certains aspects de la confidentialité, préalable absolu en relation d'aide, ont déjà été traités. Il est pourtant très difficile d'en établir le principe pour ensuite le respecter dans la pratique. Partout dans le monde, les hommes politiques et les groupes de citoyens appuient le signalement obligatoire et autres mesures semblables qui rendent la confidentialité impossible. Même dans les meilleures conditions, la confidentialité est difficile à protéger puisque des dossiers écrits ou d'autres formes de rapports sont nécessaires. On doit comprendre que dans certaines cultures, une personne qui se soustrait à l'obligation de divulguer un problème à un confident désigné ou à un membre de sa famille peut se rendre coupable d'une grave atteinte aux normes et aux attentes.

Une fois la formation reçue, les chances de conserver la confidentialité dans l'aide par les pairs - qui se fait souvent de personne à personne - peuvent être bonnes. Il est plus difficile de le faire dans les groupes d'entraide. Les responsables doivent inculquer une certaine éthique de la confidentialité de groupe à ceux pour qui une expérience de groupe ou le principe même de la confidentialité sont, à toutes fins utiles, étrangers.

Le responsable d'un groupe d'entraide doit aider les membres à comprendre les dangers de la divulgation d'informations personnelles à l'extérieur du groupe. Même si un membre ne donne pas son nom, 
certains renseignements peuvent malencontreusement servir à l'identifier, surtout dans les petites communautés.

\section{Aide par les pairs}

Cette méthode peut être utile aux personnes inquiètes d'être infectées, aux séropositifs et aux sidéens. Il est important que le conseiller et la personne qui consulte aient une préoccupation ou un problème commun, c'est-à-dire la séropositivité. Les rencontres d'aide par les pairs devraient avoir lieu dans un centre de soins de santé ou autre lieu désigné. Même si ces rencontres peuvent être assez informelles, elles ne devraient pas se tenir à la résidence du conseiller ou en des endroits publics. Il est important que la sélection du lieu où se fait l'aide par les pairs renforce l'idée qu'elle est une partie intégrante d'un système global de soins.

Le rôle le plus important du conseiller est ici d'écouter et d'aider une autre personne à exprimer ses peurs et ses inquiétudes, d'intervenir en utilisant le reflet, et d'évaluer les options possibles en vue d'une action ou d'une solution. Lorsqu'il s'agit d'un problème d'infection par le $\mathrm{VIH}$, il est impossible de dire à quelqu'un : "Je sais ce que tu ressens ". Un individu qui a vécu la même expérience (par exemple être informé d'un résultat positif à un test) peut, cependant, être plus en mesure que toute autre personne de fournir une réponse authentique, empathique et d'offrir un soutien. Il est important que l'aidant ne compare pas sa propre méthode de solution du problème à celle de la personne qui consulte. Le conseiller devrait avoir une formation qui lui permette d'utiliser des techniques et des méthodes telles que l'écoute active, le soutien émotionnel, l'établissement de liens entre les ressources sociales et celles de la famille, le reflet, le soutien à l'expression d'affliction et de deuil, et la planification de l'avenir. Les différences marquantes entre l'aide par les pairs et les autres formes d'aide sont la similitude de l'expérience vécue par les deux personnes et l'absence d'attentes relatives à la subjectivité du conseiller. Cette subjectivité contrôlée est le principal outil dont il dispose.

\section{Groupes d'entraide}

Les groupes d'entraide, de même que l'aide par les pairs, sont formés de personnes qui partagent une expérience et des peurs communes. Le groupe d'entraide peut être mené par un intervenant professionnel ou déjà formé à une aide par les pairs. Les groupes d'entraide ne devraient jamais être mis sur pied ni fonctionner sans avoir à leur tête 
une personne formée et clairement désignée. Cette dernière doit avoir vécu une formation de base dans les secteurs suivants: dynamique de groupe, confidentialité de groupe et énoncé d'un contrat de groupe, attitudes culturelles liées à la divulgation de situations privées, établissement de frontières pour un groupe.

On doit appuyer de nouveau sur la confidentialité. Les groupes d'entraide peuvent être efficaces seulement lorsqu'il existe une confiance mutuelle entre les membres de même qu'entre ceux-ci et le responsable. Des contrats explicites de confidentialité et d'aide mutuelle doivent être établis à la première session et fréquemment révisés.

Lorsqu'un groupe est mis sur pied, on doit déterminer si le nombre de membres en sera limité ou illimité. Dans un groupe ouvert, de nouveaux membres peuvent s'ajouter à n'importe quel moment tandis qu'un groupe fermé conserve le même nombre de participants aussi longtemps qu'il existe. Les groupes formés de personnes séropositives et atteintes du sida ne peuvent être ouverts à cause de la stigmatisation dont elles sont l'objet. Les membres en font partie pour une période précise pendant laquelle aucun nouveau participant n'est admis. Les groupes ouverts peuvent aider à solutionner d'autres problèmes mais la liberté d'adhésion demeure un facteur négatif dans une situation où la plus grande confidentialité est requise.

On doit aussi tenir compte d'une autre variable, soit l'hétérogénéité par rapport à l'homogénéité. Les groupes d'entraide axés sur la séropositivité ou le sida doivent être homogènes: tous les membres doivent être porteurs de l'un ou de l'autre. Par ailleurs, les sympathisants, tout comme les séropositifs et les autres, devraient former des groupes séparés et homogènes. Même si les hommes homosexuels ou hétérosexuels peuvent éprouver les mêmes réactions à l'infection ou à la maladie, leurs cadres de référence et le contexte dans lequel ils vivent sont sensiblement différents et l'on ne devrait pas les intégrer à un même groupe. Les règles qui portent sur les relations à l'extérieur du groupe et pendant les rencontres devraient être discutées et acceptées dès le départ. Le groupe permet aux participants de s'identifier aux autres, de comprendre la nature universelle de certaines de leurs émotions et de s'accorder une aide mutuelle. Ce dernier point est extrêmement important, surtout pour les personnes malades et faibles qui se sentent inutiles ou qui ne jouent aucun des rôles auxquels elles étaient habituées. Les groupes d'entraide leur permettent de se sentir utiles et de recevoir en retour le soutien émotionnel dont elles ont besoin. 


\section{Engagement et formation de liens affectifs}

Le responsable du groupe doit avoir reçu une formation qui lui permette d'utiliser les caractéristiques de la personnalité et l'expérience des membres pour favoriser l'engagement des nouveaux arrivants. Par engagement, on entend la volonté d'une personne à risquer une alliance avec une autre personne, processus qui est suivi du sentiment que l'autre la comprend réellement et est digne de confiance. L'engagement conduit à la "formation de liens ", concept qui renvoie, dans ces deux méthodes, à une fusion des états émotionnels des membres et du groupe et qui résulte en un attachement de chacun à tous.

Il sera vite évident aux travailleurs sociaux, aux éducateurs en santé et aux aidants que certains procédés visant à favoriser l'engagement et la création de liens peuvent conduire à de possibles dommages. Si l'on ne tient pas compte de la valeur de l'expérience personnelle d'un participant mais qu'on la discrédite en faveur de celle d'une autre personne par exemple, ce participant quittera le groupe dans un état pire qu'à son arrivée. Le conseiller (dans une aide par les pairs) ou le responsable d'un groupe d'entraide doivent donc être en mesure de faire une juste évaluation des méthodes de soutien auprès des participants qui se sentent délaissés ou attaqués. Ils doivent aussi développer des habiletés à fixer des limites lorsque le groupe ne fournit plus l'aide ou devient même nuisible.

\section{Conclusion}

L'aide par les pairs et les groupes d'entraide ne peuvent remplacer les services officiels de soins de santé, d'éducation en soins de santé et d'aide psychosociale. De plus, la formation appropriée de ces conseillers et responsables est un préalable à la mise en place de ces formes d'aide. Lorsque les coutumes, la tradition et la culture le permettent, chacune de ces formules peut être efficace pour suppléer à l'aide professionnelle ou la compléter. L'émergence des effets de la pandémie de VIH provoquera un engorgement de tous les systèmes officiels de santé et d'aide psychosociale. Ces méthodes seront des composantes nécessaires et vitales aux stratégies de prévention, de contrôle et d'aide, et devront être intégrées à l'ensemble du système de soins.

\section{Note}

* Traduit par madame Charlotte Gagné. 